\title{
TITLE:
}

\section{Contact-Force Control of a Flexible Timoshenko Arm}

$\operatorname{AUTHOR}(\mathrm{S})$ :

Endo, Takahiro; Sasaki, Minoru; Matsuno, Fumitoshi

CITATION:

Endo, Takahiro ...[et al]. Contact-Force Control of a Flexible

Timoshenko Arm. IEEE Transactions on Automatic Control 2017, 62(2): 1004-1009

ISSUE DATE:

2017-02

URL:

http://hdl.handle.net/2433/232623

\section{RIGHT:}

(c) 2017 IEEE. Personal use of this material is permitted. Permission from IEEE must be obtained for all other uses, in any current or future media, including reprinting/republishing this material for advertising or promotional purposes,

creating new collective works, for resale or redistribution to servers or lists, or reuse of any copyrighted component of this work in other works.; This is not the published version. Please cite only the published version.; この論文は出版社版 でありません。引用の際には出版社版をご確認ご利用ください。 


\title{
Contact-Force Control of a Flexible Timoshenko Arm
}

\author{
Takahiro Endo, Minoru Sasaki, and Fumitoshi Matsuno
}

\begin{abstract}
This paper discusses a contact-force control problem for a flexible arm. This flexible arm includes a Timoshenko beam, and thus we call it the flexible Timoshenko arm. The aim of the force control is to control the contact force at the contact point. To solve this problem, we propose a simple boundary controller and show the exponential stability of the closed-loop system by the frequency domain method. Finally, we describe simulation results carried out to investigate the validity of the proposed controller for the force control problem.
\end{abstract}

Index Terms - Flexible arm, Timoshenko beam, Distributed parameter systems, Exponentially stable.

\section{INTRODUCTION}

The dynamics of a flexible arm are expressed by partial differential equations (PDEs), which present the dynamics of the elastic link, and ordinary differential equations (ODEs), which present the dynamics of the actuators, tip load and others. Thus, the flexible arm can be represented by a hybrid PDE-ODE system. For the dynamics of flexible arms, the Timoshenko beam is widely used to represent the dynamics of the elastic link, and we describe such arms as flexible Timoshenko arms. If we focus attention on a flexible Timoshenko arm represented by the hybrid PDE-ODE system, there are several relevant previous studies [1]-[9].

These studies mainly dealt with vibration control, but vibration control alone is insufficient if the goal is to use the flexible arm for more complex tasks. It is also important to control the contact force that the end-effector of the flexible arm exerts on an object or the environment [10]. In this paper, we focus on a force control problem of a one-link flexible Timoshenko arm. In particular, we propose a simple boundary controller without any finite dimensional approximation.

Contact-force control of a flexible arm based on the infinite dimensional model has been studied previously [11]-[18]. In these studies [11]-[15], the force control problem for a one-link flexible arm was modeled by Euler-Bernoulli beams, and asymptotic/exponential stabilizing controllers were proposed. Other studies [16]-[18] discussed cooperative or grasping tasks (which are typical tasks of force control) by multiple flexible arms also modeled by Euler-Bernoulli beams. However, to the best of our knowledge, there has not yet been a study of force control for a flexible Timoshenko arm based on the infinite dimensional model. In contrast to the Euler-Bernoulli beam, the Timoshenko beam includes the effects of shear and rotation, and thus the Timoshenko beam is modified for a non-slender

This work was supported in part by JSPS KAKENHI Grant Number 23700143.

T. Endo and F. Matsuno are with the Department of Mechanical Engineering and Science, Kyoto University, Kyoto, Japan (e-mail: endo@me.kyoto-u.ac.jp, matsuno@me.kyoto-u.ac.jp).

M. Sasaki is with the Department of Mechanical Engineering, Gifu University, Gifu, Japan (e-mail: sasaki@gifu-u.ac.jp). beam and high-frequency response. Therefore, the Timoshenko beam has a wider application range than the Euler-Bernoulli beam [19], [20]. From this point of view, the contact-force control problem of the flexible Timoshenko arm is a challenging and important one, and thus we propose a simple, easy to implement boundary controller to solve the contact-force control problem of the flexible Timoshenko arm.

The paper is organized as follows. In Section II, we describe the mathematical model of a constrained one-link flexible Timoshenko arm. Further, we formulate the contact-force control problem, and propose a simple boundary controller. The semigroup setting of the closed-loop system is described in Section III, and its exponential stability is proved in Section IV. The simulation results that are described in Section V demonstrate the validity of the proposed boundary controller. Finally, Section VI presents our conclusions.

\section{DESCRIPTION OF THE PROBLEM}

\section{A. Dynamics of a constrained flexible Timoshenko arm}

Fig. 1 shows a constrained one-link flexible Timoshenko arm. One end of the arm is clamped to control actuators consisting of the rotational motor and the translational slider, and the other end has a concentrated tip mass $m$. The tip mass makes contact with the surface of an object. The flexible arm moves in the $X Y$ plane in Fig. 1; it is not affected by the acceleration of gravity. The flexible arm, with length $l$, mass per unit length $\rho$, mass moment of inertia $I_{\rho}$, cross sectional area $A$, area moment of inertia $I$, Young's modulus $E$, shear modulus $G$, and shear coefficient $\kappa$, satisfies the Timoshenko beam hypothesis.

In Fig. 1, $X Y$ is an absolute coordinate system and $x y$ is a local coordinate system, whose origin is fixed at the rotor of the motor. In addition, $x y$ translates with the slider in the $Y$-direction and rotates with the rotor of the motor. Let $J, \tau_{m}(t)$, $\theta(t), M, F_{s}(t)$, and $s(t)$ be the inertia moment of the motor, the torque by the motor, the angle of the motor, the mass of the slider, the force by the slider, and the position of the slider, respectively. Further, let $w(x, t)$ and $\phi(x, t)$ be the transverse displacement of the arm at time $t$ and spatial point $x$, and the rotation of the cross section due to bending deformation, respectively. Note that $w(x, t), \phi(x, t), \theta(t)$, and $s(t)$ are assumed to be small.

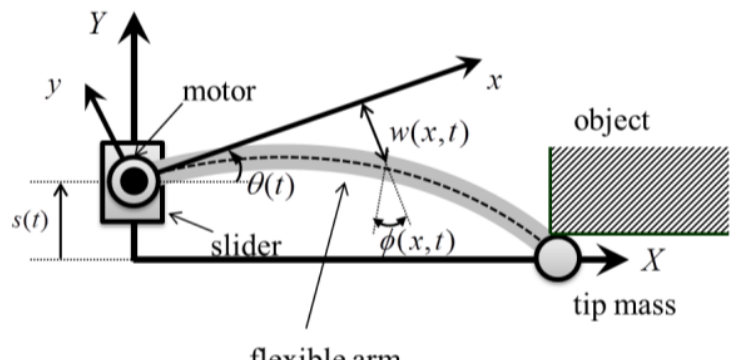

flexible arm

Fig. 1. Flexible Timoshenko arm making contact with an object. 
There is a linear actuator (slider) at the root of the arm in addition to the rotary actuator (motor); that is, the motor is installed on the slider. We use two actuators for the following reason: in vibration control of a Timoshenko beam with a fixed-free boundary, it is known that a system with one control actuator at the free end is exponentially stabilized if and only if a physically impossible condition (equal wave speeds) holds [21]. In keeping with this fact, we use two actuators here.

Since the tip mass makes contact with the surface of the object, we obtain the following geometric constraint: $\Phi \equiv l \theta(t)+w(l, t)+s(t)=0$. This constraint means that the $Y$-axis position of the tip mass is constrained on the surface of the object. The kinetic energy $E_{k}$ and the potential energy $E_{p}$ of the overall system are given by the following:

$$
\begin{aligned}
2 E_{K}= & \rho \int_{0}^{l}[x \dot{\theta}(t)+\dot{w}(x, t)+\dot{s}(t)]^{2} \mathrm{~d} x+J \dot{\theta}^{2}(t)+M \dot{s}^{2}(t) \\
& +I_{\rho} \int_{0}^{l}[\dot{\theta}(t)+\dot{\phi}(x, t)]^{2} \mathrm{~d} x+m[l \dot{\theta}(t)+\dot{w}(l, t)+\dot{s}(t)]^{2}, \\
2 E_{P}= & E I \int_{0}^{l}\left[\phi^{\prime}(x, t)\right]^{2} \mathrm{~d} x+K \int_{0}^{l}\left[\phi(x, t)-w^{\prime}(x, t)\right]^{2} \mathrm{~d} x,
\end{aligned}
$$

where $K=\kappa G A$, a dot denotes the time derivative, and a prime denotes the partial derivative with respect to $x$. Here the virtual work is given by $\delta W=\tau_{m}(t) \delta \theta(t)+F_{s}(t) \delta s(t)$.

Under the above preparation, we can obtain the following equations of motion by applying Hamilton's principle and Lagrange's multiplier, and using the procedure in [14]:

$$
\left\{\begin{array}{l}
\rho[\ddot{w}(x, t)+x \ddot{\theta}(t)+\ddot{s}(t)]+K\left[\phi^{\prime}(x, t)-w^{\prime \prime}(x, t)\right]=0, \\
I_{\rho}[\ddot{\phi}(x, t)+\ddot{\theta}(t)]+K\left[\phi(x, t)-w^{\prime}(x, t)\right]-E I \phi^{\prime \prime}(x, t)=0, \\
w(0, t)=\phi(0, t)=\phi^{\prime}(l, t)=l \theta(t)+w(l, t)+s(t)=0, \\
M \ddot{s}(t)=F_{s}(t)+K w^{\prime}(0, t) \equiv F(t), \\
J \ddot{\theta}(t)=\tau_{m}(t)+E I \phi^{\prime}(0, t) \equiv \tau(t),
\end{array}\right.
$$

with the algebraic relation

$\lambda(t)=K\left[w^{\prime}(l, t)-\phi(l, t)\right]$,

where $\lambda(t)$ is Lagrange's multiplier and is equivalent to the contact force, i.e., the shear force at the tip of the flexible arm, which arises in the direction along the normal vector of the constraint surface.

\section{B. Control objective and boundary controller}

The aim of this paper is to control the contact force at the tip of the flexible arm. In other words, the control objective is to construct a controller satisfying: $\lambda(t) \rightarrow \lambda_{d}, \dot{w}(x, t) \rightarrow 0, \dot{\phi}(x, t)$ $\rightarrow 0, \dot{\theta}(t) \rightarrow 0, \dot{s}(t) \rightarrow 0$, where $\lambda_{d}$ is the constant desired contact force. At the desired equilibrium point $\left(\lambda(t)=\lambda_{d}\right.$, $\dot{w}(x, t)=\dot{\phi}(x, t)=\dot{\theta}(t)=\dot{s}(t)=0), w(x, t)$ and $\phi(x, t)$ become the function of $x$, and $\theta(t)$ and $s(t)$ become constant. Thus, we describe them as $w_{d}(x), \phi_{d}(x), \theta_{d}$, and $s_{d}$, respectively. By substituting these into (1), (2), we obtain:

$$
\left\{\begin{array}{l}
w_{d}(x)=\lambda_{d} x\left(\frac{1}{K}+\frac{l x}{2 E I}-\frac{x^{2}}{6 E I}\right), \phi_{d}(x)=\frac{\lambda_{d} x}{E I}\left(l-\frac{x}{2}\right), \\
l \theta_{d}+s_{d}=-\lambda_{d} l\left(\frac{1}{K}+\frac{l^{2}}{3 E I}\right) .
\end{array}\right.
$$

In these relations, $w_{d}(x), \phi_{d}(x), \theta_{d}$, and $s_{d}$ mean a static transverse displacement, a static rotation of the cross section of the flexible arm, a static angle of the motor, and a static position of the slider in the case where the contact force is converged to the desired value, respectively. Furthermore, $\theta_{d}$ and $s_{d}$ are coupled through $\lambda_{d}$, and thus we cannot set $\theta_{d}$ and $s_{d}$ independently.

Based on these considerations, we propose a boundary controller that realizes the following:

$$
\left\{\begin{array}{l}
w(x, t) \rightarrow w_{d}(x), \dot{w}(x, t) \rightarrow 0, \phi(x, t) \rightarrow \phi_{d}(x), \dot{\phi}(x, t) \rightarrow 0, \\
\theta(t) \rightarrow \theta_{d}, \dot{\theta}(t) \rightarrow 0, s(t) \rightarrow s_{d}, \dot{s}(t) \rightarrow 0 .
\end{array}\right.
$$

For this purpose, we propose the following boundary controller:

$$
\begin{aligned}
& F(t)=\tilde{k}_{1} K\left[w^{\prime}(0, t)-w_{d}^{\prime}(0)\right]+\tilde{k}_{2} K \dot{w}^{\prime}(0, t)-\tilde{k}_{3}\left[s(t)-s_{d}\right]-\tilde{k}_{4} \dot{s}(t), \\
& \tau(t)=\tilde{k}_{5} E I\left[\phi^{\prime}(0, t)-\phi_{d}^{\prime}(0)\right]+\tilde{k}_{6} E I \dot{\phi}^{\prime}(0, t)-\tilde{k}_{7}\left[\theta(t)-\theta_{d}\right]-\tilde{k}_{8} \dot{\theta}(t),
\end{aligned}
$$

where feedback gain $\tilde{k}_{i}, i=1, \ldots, 8$, is a positive constant. In (4), the first and second terms are for the control: $w(x, t) \rightarrow w_{d}(x)$ and $\dot{w}(x, t) \rightarrow 0$, and the third and the forth terms are for the position control: $s(t) \rightarrow s_{d}$ and $\dot{s}(t) \rightarrow 0$. On the other hand, in (5), the first and second terms are for the control: $\phi(x, t) \rightarrow \phi_{d}(x)$ and $\dot{\phi}(x, t) \rightarrow 0$, and the third and the forth terms are for $\theta(t) \rightarrow \theta_{d}$ and $\dot{\theta}(t) \rightarrow 0$. In the controllers, $E I \phi^{\prime}(0, t)$ can be measured by the strain gauges, and $\theta(t)$ and $s(t)$ can be measured by the encoders. In addition, the shear force, $K w^{\prime}(0, t)$ (here note $\phi(0, t)=0$ ), can also be measured using strain gauges based on the difference method [22]. Here, if we use a speed-reference-type servo amplifier with speed feedback and the high-gain characteristic of the amplifier for the motor and slider, we can implement the controllers without the time derivatives. For more technical details, please see [23]. Therefore, we can easily implement the controller.

\section{Closed-LOOP SYSTEM}

\section{A. Semigroup setting}

Now let us introduce new variables:

$$
\left\{\begin{array}{l}
y_{1}(x, t)=\left[w(x, t)-w_{d}(x)\right]+x\left[\theta(t)-\theta_{d}\right]+\left[s(t)-s_{d}\right], \\
y_{2}(x, t)=\left[\phi(x, t)-\phi_{d}(x)\right]+\left[\theta(t)-\theta_{d}\right], \\
\eta_{1}(t)=k_{2} K\left[y_{1}^{\prime}(0, t)-y_{2}(0, t)\right]-\dot{y}_{1}(0, t)-k_{2} k_{3} y_{1}(0, t) / k_{1}, \\
\eta_{2}(t)=k_{6} E I y_{2}^{\prime}(0, t)-\dot{y}_{2}(0, t)-k_{6} k_{7} y_{2}(0, t) / k_{5},
\end{array}\right.
$$

based on the procedure described by [17]. Here, $k_{i}=\tilde{k}_{i} / M$ for $i=1, \ldots, 4$, and $k_{i}=\tilde{k}_{i} / J$, for $i=5, \ldots, 8$. Then the equations of motion become simple, the equilibrium point is moved to its origin, and the closed-loop system can be rewritten as

$\rho \ddot{y}_{1}(x, t)+K\left[y_{2}^{\prime}(x, t)-y_{1}^{\prime \prime}(x, t)\right]=0, x \in(0, l), t \geq 0$,

$I_{\rho} \ddot{y}_{2}(x, t)+K\left[y_{2}(x, t)-y_{1}^{\prime}(x, t)\right]-E I y_{2}^{\prime \prime}(x, t)=0$,

$y_{1}(l, t)=y_{2}^{\prime}(l, t)=0$,

$\dot{\eta}_{1}(t)=-k_{1} K\left[y_{1}^{\prime}(0, t)-y_{2}(0, t)\right]+k_{3} y_{1}(0, t)+D_{1} \dot{y}_{1}(0, t)$,

$\dot{\eta}_{2}(t)=-k_{5} E I y_{2}^{\prime}(0, t)+k_{7} y_{2}(0, t)+D_{2} \dot{y}_{2}(0, t)$,

with the algebraic relation $\lambda(t)-\lambda_{d}=K\left[y_{1}^{\prime}(l, t)-y_{2}(l, t)\right]$, where 
$D_{1}=k_{4}-k_{2} k_{3} / k_{1}$ and $D_{2}=k_{8}-k_{6} k_{7} / k_{5}$. Here, we introduced $y_{1}(x, t)$ and $y_{2}(x, t)$ to formulate the system as a classic hybrid control system [24]. In addition, we introduced $\eta_{1}(t)$ and $\eta_{2}(t)$ so that the closed-loop system becomes dissipative; that is, the estimate (17) holds. We arrived at this choice by trial and error. As a result, the restriction (16) was needed for the operator $A$ to become dissipative.

We wish to formulate the closed-loop system as a first order evolution equation in an appropriate Hilbert space, $H$, as in the following equation:

$\dot{z}(t)=A z(t), \quad z(0)=z_{0}$,

where $z(t)=\left(y_{1}(\cdot, t), \dot{y}_{1}(\cdot, t), y_{2}(\cdot, t), \dot{y}_{2}(\cdot, t), \eta_{1}(t), \eta_{2}(t)\right)^{T}$ is the state, and $z_{0}$ is the initial value. To do this, let us introduce the following Hilbert space as the state space:

$H=H_{0}^{1}(0, l) \times L^{2}(0, l) \times H^{1}(0, l) \times L^{2}(0, l) \times \mathrm{C}^{2}$,

where $H^{m}(0, l)$ is the usual Sobolev space of order $m, L^{2}(0, l)$ is the usual square integrable functional space, $H_{0}^{m}(0, l)$ $=\left\{u \in H^{m}(0, l): u(l)=0\right\}$, and $\mathrm{C}$ is the set of complex numbers. In the space $H$, we define the inner product as follows:

$$
\begin{aligned}
2\langle z, \hat{z}\rangle_{H}= & \rho \int_{0}^{l} v_{1} \overline{\hat{v}_{1}} \mathrm{~d} x+I_{\rho} \int_{0}^{l} v_{2} \overline{\hat{v}_{2}} \mathrm{~d} x+K \int_{0}^{l}\left(u_{2}-u_{1}^{\prime}\right) \overline{\left(\hat{u}_{2}-\hat{u}_{1}^{\prime}\right)} \mathrm{d} x \\
& +E I \int_{0}^{l} u_{2}^{\prime} \overline{\hat{u}_{2}^{\prime}} \mathrm{d} x+k_{3} u_{1}(0) \overline{\hat{u}_{1}(0)} / k_{1}+\eta_{1} \overline{\hat{\eta}_{1}} /\left(k_{1}+k_{2} D_{1}\right) \\
& +k_{7} u_{2}(0) \overline{\hat{u}_{2}(0)} / k_{5}+\eta_{2} \overline{\hat{\eta}_{2}} /\left(k_{5}+k_{6} D_{2}\right),
\end{aligned}
$$

for $z=\left(u_{1}, v_{1}, u_{2}, v_{2}, \eta_{1}, \eta_{2}\right)^{T}$, and $\hat{z}=\left(\hat{u}_{1}, \hat{v}_{1}, \hat{u}_{2}, \hat{v}_{2}, \hat{\eta}_{1}, \hat{\eta}_{2}\right)^{T} \in H$. It can be shown that $H$, together with the inner product (13), becomes a Hilbert space because we can show that the norm induced by (13) is equivalent to the standard norm in $H$ (please see the Appendix, where a part of the proof of the equivalence is given). In addition, we also define a linear operator $A: D(A) \subset H \rightarrow H$ by

$$
A z=\left[\begin{array}{c}
v_{1} \\
K\left(u_{1}^{\prime \prime}-u_{2}^{\prime}\right) / \rho \\
v_{2} \\
K\left(u_{1}^{\prime}-u_{2}\right) / I_{\rho}+E I u_{2}^{\prime \prime} / I_{\rho} \\
-k_{1} K\left[u_{1}^{\prime}(0)-u_{2}(0)\right]+k_{3} u_{1}(0)+D_{1} v_{1}(0) \\
-k_{5} E I u_{2}^{\prime}(0)+k_{7} u_{2}(0)+D_{2} v_{2}(0)
\end{array}\right] \text {, }
$$

with domain

$$
\begin{aligned}
& D(A)=\left\{z \in H^{2}(0, l) \times H^{1}(0, l) \times H^{2}(0, l) \times H^{1}(0, l) \times \mathrm{C}^{2}:\right. \\
& u_{1}(l)=v_{1}(l)=u_{2}^{\prime}(l)=0 \text {, } \\
& \eta_{1}=k_{2} K\left[u_{1}^{\prime}(0)-u_{2}(0)\right]-v_{1}(0)-k_{2} k_{3} u_{1}(0) / k_{1} \text {, } \\
& \left.\eta_{2}=k_{6} E I u_{2}^{\prime}(0)-v_{2}(0)-k_{6} k_{7} u_{2}(0) / k_{5}\right\} \text {. }
\end{aligned}
$$

The closed-loop system (6), (7), (9), (10) can then be written as the first order evolution equation (11) on $H$.

\section{B. Properties of the closed-loop system}

As the properties of the closed-loop system, we obtain the following lemma:

Lemma 1: If feedback gain $k_{i}, i=1, \ldots, 8$, satisfies

$k_{1} k_{4}>k_{2} k_{3}$, and $k_{5} k_{8}>k_{6} k_{7}$,

that is, if $D_{1}, D_{2}>0$, then the operator $A$ generates a
$C_{0}$-semigroup of contractions. Furthermore, the operator $-A^{-1}$ is compact. Therefore, the spectrum $\sigma(A)$ of the operator $A$ consists only of the isolated eigenvalues.

Proof: First, we show that the operator $A$ is dissipative. For any $z=\left(u_{1}, v_{1}, u_{2}, v_{2}, \eta_{1}, \eta_{2}\right)^{T} \in D(A)$, it follows that

$$
\begin{aligned}
& 2 \operatorname{Re}\langle A z, z\rangle_{H}=\langle A z, z\rangle_{H}+\langle z, A z\rangle_{H} \\
= & -\frac{k_{2}}{k_{1}\left(k_{1}+k_{2} D_{1}\right)}\left|k_{1} K\left[u_{1}^{\prime}(0)-u_{2}(0)\right]-k_{3} u_{1}(0)\right|^{2}-\frac{D_{1}}{k_{1}+k_{2} D_{1}}\left|v_{1}(0)\right|^{2} \\
& -\frac{k_{6}}{k_{5}\left(k_{5}+k_{6} D_{2}\right)}\left|k_{5} E I u_{2}^{\prime}(0)-k_{7} u_{2}(0)\right|^{2}-\frac{D_{2}}{k_{5}+k_{6} D_{2}}\left|v_{2}(0)\right|^{2} \leq 0 .
\end{aligned}
$$

Here we used integration by parts and the boundary conditions in (15) to obtain (17). Hence, the operator $A$ is dissipative.

Next, we show that $0 \in \rho(A)$, where $\rho(A)$ is the resolvent set of the operator $A$. For any given $\hat{z}=\left(\hat{u}_{1}, \hat{v}_{1}, \hat{u}_{2}, \hat{v}_{2}, \hat{\eta}_{1}, \hat{\eta}_{2}\right)^{T} \in H$, we find a solution $z=\left(u_{1}, v_{1}, u_{2}, v_{2}, \eta_{1}, \eta_{2}\right)^{T} \in D(A)$ of $-A z=\hat{z}$. Eliminating $v_{1}, v_{2}, \eta_{1}$, and $\eta_{2}$ in this equation, we obtain the following equations:

$$
\left\{\begin{array}{l}
K\left[u_{2}^{\prime}(x)-u_{1}^{\prime \prime}(x)\right]=\rho \hat{v}_{1}(x), \\
K\left[u_{2}(x)-u_{1}^{\prime}(x)\right]-E I u_{2}^{\prime \prime}(x)=I_{\rho} \hat{v}_{2}(x), \\
u_{1}(l)=u_{2}^{\prime}(l)=0, \quad k_{5} E I u_{2}^{\prime}(0)-k_{7} u_{2}(0)=\hat{\eta}_{2}-D_{2} \hat{u}_{2}(0), \\
k_{1} K\left[u_{1}^{\prime}(0)-u_{2}(0)\right]-k_{3} u_{1}(0)=\hat{\eta}_{1}-D_{1} \hat{u}_{1}(0) .
\end{array}\right.
$$

Now, we integrate first equation of (18) and substitute it into second equation of (18). The obtained equation then yields

$$
\begin{aligned}
u_{2}(x)= & \frac{\rho}{E I} \int_{0}^{x} \frac{(x-s)^{2}}{2} \hat{v}_{1}(s) \mathrm{d} s-\frac{I_{\rho}}{E I} \int_{0}^{x}(x-s) \hat{v}_{2}(s) \mathrm{d} s \\
& +\frac{x^{2}}{2 E I} C_{1}+C_{2} x+C_{3},
\end{aligned}
$$

where $C_{i}, i=1,2,3$, is a constant, which is determined by the boundary conditions. Further, we obtain $u_{1}(x)=$ $\rho \int_{0}^{x} \alpha[x-s] \hat{v}_{1}(s) \mathrm{d} s-I \rho \int_{0}^{x}(x-s)^{2} \hat{v}_{2}(s) \mathrm{d} s /(2 E I)+\alpha[x] C_{1}+x^{2} C_{2} / 2+C_{3} x+$ $C_{4}$, where $C_{4}$ is a constant, and $\alpha[x] \equiv x^{3} /(6 E I)-x / K$. Substituting these solutions into the remaining boundary conditions, we get the matrix form relation: $M\left[C_{1}, C_{2}, C_{3}, C_{4}\right]^{T}=\left[f_{1}, f_{2}, f_{3}, f_{4}\right]^{T}$, where $M \in \mathrm{C}^{4 \times 4}$ is a matrix and $f_{i}, i=1, \ldots, 4$, is a scalar. A straightforward calculation shows $\operatorname{det} M \neq 0$ and thus, the coefficient $C_{i}, i=1, \ldots, 4$, can be uniquely determined. The remaining unknowns $v_{1}, v_{2}, \eta_{1}$, and $\eta_{2}$ can be found using $u_{1}$ and $u_{2}$. Therefore, we could find a solution $z$, which means $0 \in \rho(A)$.

From the fact that the operator $A$ is dissipative and $0 \in \rho(A)$, that is, $\operatorname{Ran}(-A)=H$, we establish $A$ generates a $C_{0}$-semigroup of contractions by the Lumer-Phillips theorem [25].

Finally, we show that the operator $-A^{-1}$ is compact. For this, we first determine the following estimate:

$\|z\|_{H^{2} \times H^{1} \times H^{2} \times H^{1} \times \mathrm{C}^{2}} \leq C^{\prime}\|\hat{z}\|_{H}$,

where $\|\cdot\|_{H^{m}}$ is the usual norm in $H^{m}(0, l)$, and $C^{\prime}$ is a positive constant. For the coefficient $C_{i}, i=1, \ldots, 4$, we obtain the following: for $i=1, \ldots, 4$, 
$\left|C_{i}\right|^{2} \leq C_{i}^{\prime} B, \quad B=\sum_{i=1}^{2}\left(\left\|\hat{v}_{i}\right\|_{L^{2}}^{2}+\left\|\hat{u}_{i}\right\|_{L^{2}}^{2}+\left\|\hat{u}_{i}^{\prime}\right\|_{L^{2}}^{2}+\left|\eta_{i}\right|^{2}\right)$,

where $\|\cdot\|_{L^{2}}^{2}=\int_{0}^{l}|\cdot|^{2} \mathrm{~d} x, C_{i}^{\prime}, i=1, \ldots, 4$, is a positive constant; we used the Cauchy-Schwarz inequality and the following inequality to obtain (21):

$$
|a+b|^{2} \leq(|a|+|b|)^{2} \leq 2\left(|a|^{2}+|b|^{2}\right), \quad a, b \in \mathrm{C} .
$$

In addition, by using these inequalities, we also obtain the following estimates: for $i=1,2,\left\|u_{i}\right\|_{H^{2}}^{2} \leq C_{5}^{\prime} B,\left\|v_{i}\right\|_{H^{1}}^{2} \leq$ $C_{6}^{\prime}\left(\left\|\hat{u}_{i}\right\|_{L^{2}}^{2}+\left\|\hat{u}_{i}^{\prime}\right\|_{L^{2}}^{2}\right)$, and $\left|\eta_{i}\right|^{2} \leq C_{7}^{\prime} B$, where $C_{i}^{\prime}, i=5,6,7$, is some positive constant. Thus we obtain $\|z\|_{H^{2} \times H^{1} \times H^{2} \times H^{1} \times \mathrm{C}^{2}}^{2} \leq C_{8}^{\prime} B \leq C_{9}^{\prime}\|\hat{z}\|_{H}^{2}$, where $C_{i}^{\prime}, i=8,9$ is a positive constant, and we used (A1) for obtaining final estimation. Thus, the desired estimate (20) is satisfied, and the operator $-A^{-1}$ is compact by the Sobolev imbedding theorem [25].

Let $S(t)$ be a $C_{0}$-semigroup of contractions generated by the operator $A$. Then, Lemma 1 means that the closed-loop system (11) has a unique solution $z(t)=S(t) z_{0} \in D(A)$, where $z_{0} \in D(A)$.

Further, $y_{1}$ and $y_{2} \in H^{2}(0, l)$ in the solution, and thus the contact force $\lambda(t)-\lambda_{d}=K\left[y_{1}^{\prime}(l, t)-y_{2}(l, t)\right]$ also exists.

\section{EXPONENTIAL STABILITY}

We investigate the exponential stability of the closed-loop system. Since we could not find the Lyapunov functional to show the exponential stability, we consider exponential stability using the frequency domain method. Here, note that although there is another powerful approach to prove the exponential stability (i.e., the Riesz basis approach [26]), we use the frequency domain method where the calculation is easy. According to the frequency domain method, we need to show the following two facts to prove the exponential stability of a $C_{0}$-semigroup of contractions in a Hilbert space [27]:

(i) $\rho(A) \supset\{i \beta: \beta \in \mathrm{R}\} \equiv i \mathrm{R}$,

(ii) $\varlimsup_{|\beta| \rightarrow \infty}\left\|(\mathrm{i} \beta-A)^{-1}\right\|_{H}<\infty$.

Here, if a $C_{0}$-semigroup of contractions in a Hilbert space satisfies (23) and (24), the exponential stability of the $C_{0}$-semigroup is obtained from the well-known Gearhart-Prüss-Greiner theorem [28]. In the following, we demonstrate fact (i) in Lemma 2, and (ii) in Lemma 3.

Lemma 2: Assume that the feedback gain $k_{i}, i=1, \ldots, 8$, satisfies (16). Then, $i \mathrm{R} \subset \rho(A)$.

Proof: We show that the spectrum $\sigma(A)$ consists only of the isolated eigenvalues in Lemma 1 . Thus, to prove that the imaginary axis belongs to the resolvent set $\rho(A)$, we need to show that there are no eigenvalues on the imaginary axis.

Let $s=i \beta$ and $\phi=\left[\phi_{1}, \phi_{2}, \phi_{3}, \phi_{4}, \phi_{5}, \phi_{6}\right]^{T} \in D(A)$ be an eigenvalue and the corresponding eigenfunction of the operator $A$, respectively, where $\beta \in \mathrm{R}$. Now let us consider the eigenvalue problem $A \phi=s \phi$. Here, we have shown that $0 \in \rho(A)$, and thus $\beta \neq 0$. Then, we can obtain $\operatorname{Re}\langle A \phi, \phi\rangle_{H}=0$, and this means the following from (17):

$\left\{\begin{array}{l}k_{1} K\left[\phi_{1}^{\prime}(0)-\phi_{3}(0)\right]-k_{3} \phi_{1}(0)=0, \phi_{2}(0)=0, \\ k_{5} E I \phi_{3}^{\prime}(0)-k_{7} \phi_{3}(0)=0, \phi_{4}(0)=0 .\end{array}\right.$

Eliminating $\phi_{2}, \phi_{4}, \phi_{5}$, and $\phi_{6}$ in the equation $A \phi=s \phi$, and accepting the fact of (25) gives the followings:

$\phi_{1}^{\prime \prime}(x)-\phi_{3}^{\prime}(x)-a_{1} \phi_{1}(x)=0, \phi_{3}^{\prime \prime}(x)-a_{2} \phi_{3}(x)-a_{3} \phi_{1}^{\prime}(x)=0$,

$\phi_{1}(0)=\phi_{1}(l)=\phi_{1}^{\prime}(0)=\phi_{3}(0)=\phi_{3}^{\prime}(0)=\phi_{3}^{\prime}(l)=0$,

where $a_{1}=\rho s^{2} / K, a_{2}=\left(I_{\rho} s^{2}+K\right) /(E I)$, and $a_{3}=-K /(E I)$. It is easy to see that the solutions of (26) are $\phi_{1}=\phi_{3}=0$. From this, we can see that the eigenvalue problem $A \phi=s \phi$ has only a zero solution, $\phi=0$. This contradicts the fact that the $\phi$ is an eigenfunction, and thus the proof is completed.

Lemma 3: Assume that the feedback gain $k_{i}, i=1, \ldots, 8$, satisfies (16). Then, (24) holds.

Proof: To prove (24), we use the contradiction argument method developed in [27]. According to this method, if (24) is false, then there exists a sequence $\beta_{n} \in \mathrm{R}$ with $\beta_{n} \rightarrow \infty$ and a sequence $z_{n} \in D(A)$ with $\left\|z_{n}\right\|_{H}=1$ such that:

$\left(i \beta_{n}-A\right) z_{n} \equiv \phi_{n} \rightarrow 0$ in $H$,

where $z_{n}=\left[u_{1 n}, v_{2 n}, u_{3 n}, v_{4 n}, \eta_{1 n}, \eta_{2 n}\right]^{T}, \phi_{n}=\left[\phi_{1 n}, \phi_{2 n}, \phi_{3 n}, \phi_{4 n}, \phi_{5 n}, \phi_{6 n}\right]^{T}$.

Here, (27) with (A1) means the following:

$i \beta_{n} u_{1 n}-v_{1 n}=\phi_{1 n}, i \beta_{n} v_{1 n}+K\left(u_{2 n}^{\prime}-u_{1 n}^{\prime \prime}\right) / \rho=\phi_{2 n}$,

$i \beta_{n} u_{2 n}-v_{2 n}=\phi_{3 n}, i \beta_{n} v_{2 n}+K\left(u_{2 n}-u_{1 n}^{\prime}\right) / I_{\rho}-E I u_{2 n}^{\prime \prime} / I_{\rho}=\phi_{4 n}$,

$i \beta_{n} \eta_{1 n}+k_{1} K\left[u_{1 n}^{\prime}(0)-u_{2 n}(0)\right]-k_{3} u_{1 n}(0)-D_{1} v_{1 n}(0)=\phi_{5 n}$,

$i \beta_{n} \eta_{2 n}+k_{5} E I u_{2 n}^{\prime}(0)-k_{7} u_{2 n}(0)-D_{2} v_{2 n}(0)=\phi_{6 n}$,

$\int\left\|\phi_{2 n}\right\|_{L^{2}} \rightarrow 0,\left\|\phi_{3 n}^{\prime}\right\|_{L^{2}} \rightarrow 0,\left\|\phi_{4 n}\right\|_{L^{2}} \rightarrow 0,\left\|\phi_{3 n}-\phi_{1 n}^{\prime}\right\|_{L^{2}} \rightarrow 0$,

$\left\{\left|\phi_{1 n}(0)\right| \rightarrow 0,\left|\phi_{3 n}(0)\right| \rightarrow 0,\left|\phi_{5 n}\right| \rightarrow 0,\left|\phi_{6 n}\right| \rightarrow 0\right.$,

$\left\|\phi_{1 n}\right\|_{L^{2}} \rightarrow 0,\left\|\phi_{1 n}^{\prime}\right\|_{L^{2}} \rightarrow 0,\left\|\phi_{3 n}\right\|_{L^{2}} \rightarrow 0$.

Now we show the contradictions of $\left\|z_{n}\right\|_{H}=1$; in other words, we demonstrate that $\left\|z_{n}\right\|_{H} \rightarrow 0$.

From (17) and (27), we obtain

$\left\{\left|k_{1} K\left[u_{1 n}^{\prime}(0)-u_{2 n}(0)\right]-k_{3} u_{1 n}(0)\right| \rightarrow 0,\left|v_{1 n}(0)\right| \rightarrow 0\right.$,

$\left\{\left|k_{5} E I u_{2 n}^{\prime}(0)-k_{7} u_{2 n}(0)\right| \rightarrow 0,\left|v_{2 n}(0)\right| \rightarrow 0\right.$.

Here, using (22) and (34) in $\eta_{1 n}$ and $\eta_{2 n}$ in (15) leads to

$\left|\eta_{\text {in }}\right| \rightarrow 0, \quad i=1,2$.

On the other hand, from first equations in (28) and (29), (34), and the fact that $\left|\beta_{n}\right|$ is bounded below from zero, we have

$\left|u_{\text {in }}(0)\right| \rightarrow 0, \quad i=1,2$.

Furthermore, (34) with (36) and (22) yields

$\left|u_{\text {in }}^{\prime}(0)\right| \rightarrow 0, \quad i=1,2$.

Now, eliminating $v_{1 n}$ in (28), then multiplying the obtained equation by $\rho(x-l) \overline{u_{1 n}^{\prime}}$ and integrating it from 0 to $l$ yields $\int_{0}^{l}\left[\rho \beta_{n}^{2} u_{1 n}-K\left(u_{2 n}^{\prime}-u_{1 n}^{\prime \prime}\right)\right](x-l) \overline{u_{1 n}^{\prime}} \mathrm{d} x=-\rho \int_{0}^{l}\left(i \beta_{n} \phi_{1 n}+\phi_{2 n}\right)(x-l) \overline{u_{1 n}^{\prime}} \mathrm{d} x$. 
Using the integration by parts, (36), and the Cauchy-Schwarz inequality, the right-hand side of this equation can be rewritten as follows:

$$
\begin{aligned}
& -\rho \int_{0}^{l}\left(i \beta_{n} \phi_{1 n}+\phi_{2 n}\right)(x-l) \overline{u_{1 n}^{\prime}} \mathrm{d} x \\
& \quad \leq C_{1}^{\prime \prime}\left[\left\|\phi_{1 n}\right\|_{L^{2}}+\left\|\phi_{1 n}^{\prime}\right\|_{L^{2}}\left\|\beta_{n} u_{1 n}\right\|_{L^{2}}+C_{2}^{\prime \prime}\left\|\phi_{2 n}\right\|_{L^{2}}\left\|u_{1 n}^{\prime}\right\|_{L^{2}},\right.
\end{aligned}
$$

where $C_{1}^{\prime \prime}$ and $C_{2}^{\prime \prime}$ are constants. Furthermore, from the first equation in (28), (33), and the boundedness of $\left\|z_{n}\right\|_{H}$, that is, $\left\|z_{n}\right\|_{H}=1$, and (A1), $\left\|\beta_{n} u_{1 n}\right\|_{L^{2}}$ and $\left\|u_{1 n}^{\prime}\right\|_{L^{2}}$ are bounded. Thus, from (32) and (33) we obtain

$$
\int_{0}^{l}\left[\rho \beta_{n}^{2} u_{1 n}-K\left(u_{2 n}^{\prime}-u_{1 n}^{\prime \prime}\right)\right](x-l) \overline{u_{1 n}^{\prime}} \mathrm{d} x \rightarrow 0 .
$$

Here, a simple calculation gives

$$
\begin{aligned}
& \operatorname{Re} \int_{0}^{l}\left[\rho \beta_{n}^{2} u_{1 n}-K\left(u_{2 n}^{\prime}-u_{1 n}^{\prime \prime}\right)\right](x-l) \overline{u_{1 n}^{\prime}} \mathrm{d} x \\
& \quad \rightarrow-\frac{\rho}{2}\left\|\beta_{n} u_{1 n}\right\|_{L^{2}}^{2}-\operatorname{Re} \int_{0}^{l} K(x-l) u_{2 n}^{\prime} \overline{u_{1 n}^{\prime}} \mathrm{d} x-\frac{K}{2}\left\|u_{1 n}^{\prime}\right\|_{L^{2}}^{2},
\end{aligned}
$$

using (36) and (37). Therefore, we obtain the following from (38) and (39):

$$
-\rho\left\|\beta_{n} u_{1 n}\right\|_{L^{2}}^{2}-2 \operatorname{Re} \int_{0}^{l} K(x-l) u_{2 n}^{\prime} \overline{u_{1 n}^{\prime}} \mathrm{d} x-K\left\|u_{1 n}^{\prime}\right\|_{L^{2}}^{2} \rightarrow 0 .
$$

Similarly, if we eliminate $v_{2 n}$ in (30), multiply the obtained equation by $I_{\rho}(x-l) \overline{u_{2 n}^{\prime}}$, and integrate it from 0 to $l$, then the calculations lead to:

$$
-\left(I_{\rho}-\frac{K}{\beta_{n}^{2}}\right)\left\|\beta_{n} u_{2 n}\right\|_{L^{2}}^{2}+2 \operatorname{Re} \int_{0}^{l} K(x-l) u_{2 n}^{\prime} \overline{u_{1 n}^{\prime}} \mathrm{d} x-E I\left\|u_{2 n}^{\prime}\right\|_{L^{2}}^{2} \rightarrow 0 .
$$

By taking the sum of (40) and (41), we obtain

$$
-\rho\left\|\beta_{n} u_{1 n}\right\|_{L^{2}}^{2}-K\left\|u_{1 n}^{\prime}\right\|_{L^{2}}^{2}-\left(I_{\rho}-\frac{K}{\beta_{n}^{2}}\right)\left\|\beta_{n} u_{2 n}\right\|_{L^{2}}^{2}-E I\left\|u_{2 n}^{\prime}\right\|_{L^{2}}^{2} \rightarrow 0 .
$$

Here, each coefficient is positive, and thus we obtain

$$
\left\|\beta_{n} u_{i n}\right\|_{L^{2}} \rightarrow 0,\left\|u_{i n}^{\prime}\right\|_{L^{2}} \rightarrow 0, \quad i=1,2 \text {. }
$$

Further, we also obtain the following from the first equations in (28), (29), and (43):

$\left\|v_{\text {in }}\right\|_{L^{2}} \rightarrow 0, \quad i=1,2$.

On the other hand, we first multiply the first equation in (28) by $\rho \overline{v_{1 n}}$, and the first equation in (29) by $I_{\rho} \overline{v_{2 n}}$. Then, the sum of the obtained two equations yields

$i \beta_{n} \rho \int_{0}^{l} u_{1 n} \overline{v_{1 n}} \mathrm{~d} x+i \beta_{n} I_{\rho} \int_{0}^{l} u_{2 n} \overline{v_{2 n}} \mathrm{~d} x \rightarrow 0$,

using the Cauchy-Schwarz inequality, (33), and (44). Similarly, we multiply the second equation in (28) by $\rho \overline{u_{1 n}}$ and the second equation in (29) by $I_{\rho} \overline{u_{2 n}}$. Then, the sum of the obtained two equations leads to:

$$
i \beta_{n} \rho \int_{0}^{l} v_{1 n} \overline{u_{1 n}} \mathrm{~d} x+i \beta_{n} I_{\rho} \int_{0}^{l} v_{2 n} \overline{u_{2 n}} \mathrm{~d} x+K\left\|u_{2 n}-u_{1 n}^{\prime}\right\|_{L^{2}}^{2}+E I\left\|u_{2 n}^{\prime}\right\|_{L^{2}}^{2} \rightarrow 0,
$$

using the integration by parts, the Cauchy-Schwarz inequality, $\left\|z_{n}\right\|_{H}=1$, (A1), (32), and (36). Here, by taking the sum of (45) and (46), and the real parts of the obtained estimate, we obtain the following:

$$
K\left\|u_{2 n}-u_{1 n}^{\prime}\right\|_{L^{2}}^{2}+E I\left\|u_{2 n}^{\prime}\right\|_{L^{2}}^{2} \rightarrow 0 .
$$

Finally, from (35), (36), (44), and (47), we obtain $\left\|z_{n}\right\|_{H} \rightarrow 0$, and this is the contradiction of $\left\|z_{n}\right\|_{H}=1$. Thus, the claimed is proved.

Lemma 3 and 4 are summarized in the following theorem for the exponential stability of the closed-loop system (11).
Theorem 1: Assume that the feedback gain $k_{i}, i=1, \ldots, 8$, satisfies (16). Then, the closed-loop system (11) is exponentially stable.

Proof: Lemma 3, 4, and the frequency domain method [27] leads to the exponential stability of the closed-loop system.

\section{Simulations}

Numerical simulations were conducted using Laplace transform and the numerical inverse Laplace transform method [29] with zero initial conditions. For the arm, an aluminum cylinder was used. The physical parameters were as follows: $l=1.00 \mathrm{~m}, \rho=2.54 \mathrm{~kg} / \mathrm{m}, I_{\rho}=2.83 \times 10^{-2} \mathrm{kgm}, A=9.39 \times 10^{-4} \mathrm{~m}^{2}$, $I=1.05 \times 10^{-5} \mathrm{~m}^{4}, E=69.00 \times 10^{9} \mathrm{~Pa}, G=2.57 \times 10^{10} \mathrm{~Pa}, \kappa=0.89$.

First, we considered the step responses of the desired contact force, $\lambda_{d}=-100 \mathrm{~N}$, and the desired position of the slider, $s_{d}=0.1 \mathrm{~m}$. Here, note that $\lambda_{d}=-100 \mathrm{~N}$ means that the flexible arm pushed the object by the force of $100 \mathrm{~N}$. In the simulations, we set the feedback gains $k_{i}, i=1, \ldots, 8$ as follows: $k_{1} K=k_{5} E I=1, k_{2} K=k_{6} E I=0.2, k_{3}=k_{7}=16, k_{4}=k_{8}=8$. These gains were selected so that they satisfied the conditions of (16) by trial and error.

Fig. 2 shows the simulation results of $w(x, t), \phi(x, t), s(t)$, $\theta(t)$, and $\lambda(t)$. In Fig. 2 (a) and (b), the dotted lines at $t=5$ show the desired value $w_{d}(x)$ and $\phi_{d}(x)$, respectively, and we found that $w(x, t)$ and $\phi(x, t)$ converged to the desired values. Further, in Fig. 2 (c)-(e), the solid line represents the response and the dotted line shows the desired value; we also found that $s(t), \theta(t)$, and $\lambda(t)$ converged to the desired values, that the proposed controller worked well in the step responses, and that the controller was effective for the contact force control.

Here, to implement the controller, we require the following physical parameters: $E, I, G, A, l$. Although it is easy to measure $I, A$, and $l$, it is not easy to obtain accurate $E$ and $G$. Thus, we carried out the simulation when the Young's modulus $E$ in the controller had some uncertainty, and considered the robustness

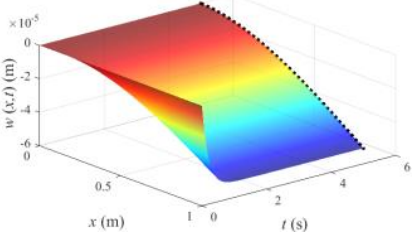

(a)

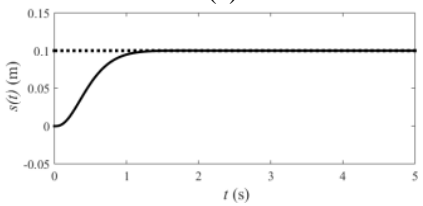

(c)

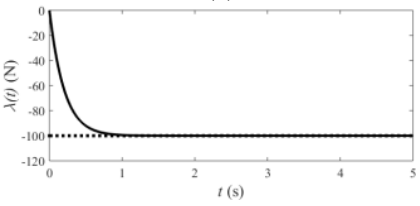

(e)

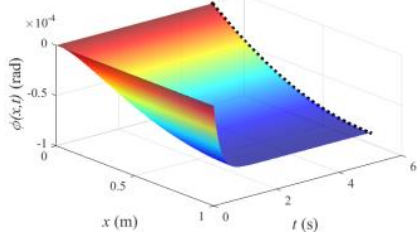

(b)

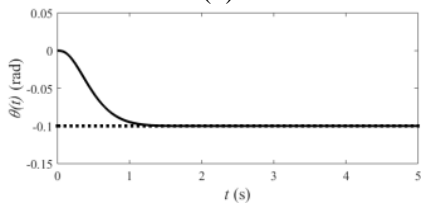

(d)

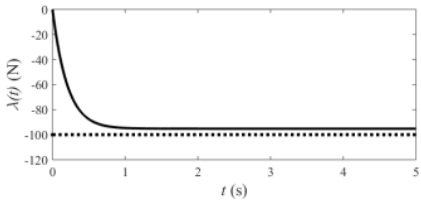

(f)
Fig. 2. Step responses. (a) $w(x, t)$; (b) $\phi(x, t)$; (c) $s(t)$; (d) $\theta(t) ;($ e) $\lambda(t)$; (f) $\lambda(t)$ when $E$ was changed. 
with respect to the physical parameter. In particular, we set $E$ as $E \times 1.05$ in the controller. The shear modulus is $G=E /(2(1+v))$, where $v$ is a Poisson's ratio. Thus, $G$ in the controller was also changed according to the change of $E$. We show the response of the $\lambda(t)$ of this simulation in Fig. 2 (f). Although the response did not diverge, the response has a steady-state error. Thus, to obtain fine responses, we need to measure accurate physical parameters before the control.

\section{CONCLUSION}

We described a contact-force control problem with regards to a constrained one-link flexible Timoshenko arm. To solve the contact force control problem of such a system, we have proposed a simple boundary controller, which is easy to implement. Furthermore, the exponential stability of the closed-loop system was proved using the frequency domain method without any finite dimensional approximation. Finally, we carried out several simulations, the results of which showed the validity of the proposed boundary controller.

While this paper addresses the boundary controller only for a contact-force control problem, force control can be extended to more complex tasks, such as cooperative tasks and grasping tasks using multiple arms. We plan to propose controllers for use in other tasks in future research.

\section{APPENDIX}

Now, we show the following estimate, which is used in the proof of Lemma 1:

$$
\left\|\left(u_{1}, u_{2}\right)\right\|_{2}^{2} \leq \gamma_{1}\left\|\left(u_{1}, u_{2}\right)\right\|_{1}^{2} \text {, }
$$

for a positive constant $\gamma_{1}$, where

$$
\left\{\begin{array}{l}
2\left\|\left(u_{1}, u_{2}\right)\right\|_{1}^{2}=E I\left\|u_{2}^{\prime}\right\|_{L^{2}}^{2}+K\left\|u_{2}-u_{1}^{\prime}\right\|_{L^{2}}^{2}+k_{3}\left|u_{1}(0)\right|^{2} / k_{1}+k_{7}\left|u_{2}(0)\right|^{2} / k_{5}, \\
\left\|\left(u_{1}, u_{2}\right)\right\|_{2}^{2}=\left\|u_{1}\right\|_{L^{2}}^{2}+\left\|u_{1}^{\prime}\right\|_{L^{2}}^{2}+\left\|u_{2}\right\|_{L^{2}}^{2}+\left\|u_{2}^{\prime}\right\|_{L^{2}}^{2},
\end{array}\right.
$$

for $\left(u_{1}, u_{2}\right) \in H_{0}^{1}(0, l) \times H^{1}(0, l)$. Here, note that $u_{i}(x)=\int_{0}^{x} u_{i}^{\prime}(x) \mathrm{d} x+$

$u_{i}(0)$ for $i=1,2$. From these equations, the Cauchy-Schwarz

inequality and inequality (22), we obtain

$\left\|u_{i}\right\|_{L^{2}}^{2} \leq \gamma_{2}\left(\left\|u_{i}^{\prime}(x)\right\|_{L^{2}}^{2}+\left|u_{i}(0)\right|^{2}\right)$,

where $\gamma_{2}$ is a positive constant. On the other hand, note the following: $\left|u_{2}-u_{1}^{\prime}\right|^{2}=\left|u_{2}\right|^{2}-2 \operatorname{Re}\left(u_{2} \overline{u_{1}^{\prime}}\right)+\left|u_{1}^{\prime}\right|^{2}$. Integrating this equation and using the inequality $2|a||b| \leq \delta|a|^{2}+|b|^{2} / \delta$ for $a, b \in \mathrm{C}, \delta \in \mathrm{R}, \delta>0$, gives

$\left\|u_{2}-u_{1}^{\prime}\right\|_{L^{2}}^{2} \geq(1-\delta)\left\|u_{2}\right\|_{L^{2}}^{2}+(1-1 / \delta)\left\|u_{1}^{\prime}\right\|_{L^{2}}^{2}$.

Therefore, using (A2) and (A3), we obtain

$\left\|\left(u_{1}, u_{2}\right)\right\|_{1}^{2} \geq \gamma_{3}\left(\left\|u_{2}\right\|_{L^{2}}^{2}+\left\|u_{1}^{\prime}\right\|_{L^{2}}^{2}\right)$,

where $\gamma_{3}=\min \left\{\gamma_{4}, \gamma_{5}\right\}, \quad \gamma_{4}=\min \left\{E I / 2, k_{7} /\left(2 k_{5}\right)\right\} / \gamma_{2}+K(1-\delta) / 2$, $\gamma_{5}=K(1-1 / \delta) / 2$, and we set $1<\delta<1+2 \min \left\{E I / 2, k_{7} /\left(2 k_{5}\right)\right\} /\left(K \gamma_{2}\right)$. Thus, we obtain (A1) by using (A2) and (A4).

\section{REFERENCES}

[1] Ö. Morgül, "Dynamic Boundary Control of the Timoshenko Beam," Automatica, vol.28, no.6, pp.1255-1260, 1992.

[2] F. Zhang, D.M. Dawson, M.S. de Queiroz, and P. Vedagarbha, "Boundary Control of the Timoshenko Beam with Free-End Mass/Inertial Dynamics," In Proc. IEEE CDC, pp.245-250, 1997.
[3] S.W. Taylor and S.C.B. Yau, "Boundary control of a rotating Timoshenko beam," ANZIAM J., vol.44, pp.E143-E184, 2003.

[4] H. Rastgoftar, M. Mahmoodi, M. Eghtesad, and M. Kazemi, "Stability analysis of a flexible two-link Timoshenko manipulator using boundary control method," In Proc. ASME IMECE, pp.409-415, 2008.

[5] M. Grobbelaar-Van Dalsen, "Uniform stability for the Timoshenko beam with tip load," J. Math. Anal. Appl., vol.361, no.2, pp.392-400, 2010.

[6] Z.J. Han and G.Q. Xu, "Dynamical behavior of a hybrid system of nonhomogeneous timoshenko beam with partial non-collocated inputs," $J$. Dyn. Control Syst., vol.17, no.1, pp.77-121, 2011.

[7] M. Sasaki, T. Ueda, Y. Inoue, and W. J. Book, "Passivity-Based Control of Rotational and Translational Timoshenko Arms," Advances in Acoustics and Vibration, vol.2012, Article ID 174816, 6 pages, 2012.

[8] W. He, S. Zhang, and S.S. Ge, "Boundary Output-Feedback Stabilization of a Timoshenko Beam Using Disturbance Observer," IEEE Trans. Ind. Electron., vol.60, no.11, pp.5186-5194, 2013.

[9] H. Ramírez, Y. Le Gorrec, A. Macchelli, and H. Zwart, "Exponential Stabilization of Boundary Controlled Port-Hamiltonian Systems With Dynamic Feedback," IEEE Trans. Autom. Control, vol.59, no.10, pp.2849-2855, 2014.

[10] L. Villani and J. De Schutter, "Force Control," in Handbook of Robotics, B. Siciliano and O. Khatib, eds. Springer, pp.161-185, ch. 7, 2008.

[11] Y. Morita, F. Matsuno, Y. Kobayashi, M. Ikeda, H. Ukai, and H. Kando, "Lyapunov-based force control of a flexible arm considering bending and torsional deformation," In Proc. IFAC World Congress, pp.832-832, 2002.

[12] F.M. Ching and D. Wang, "Exact solution and infinite-dimensional stability analysis of a single flexible link in collision," IEEE Trans. Robot. Autom. Vol.19, no.6, pp.1015-1020, 2003.

[13] Y. Shen, N. Xi, U.C. Wejinya, W.J. Li, and J. Xiao, "Infinite dimensional system approach for hybrid force/position control in micromanipulation," In Proc. IEEE ICRA, pp.2912-2917, 2004.

[14] T. Endo, F. Matsuno, and H. Kawasaki, "Force Control and Exponential Stabilisation of One-Link Flexible Arm," Int. J. Control, vol.87, no.9, pp.1794-1807, 2014.

[15] T. Endo and H. Kawasaki, "Bending Moment-based Force Control of Flexible Arm Under Gravity," Mechanism and Machine Theory, vol.79, pp.217-229, 2014

[16] F. Matsuno, A. Hayashi, "PDS cooperative control of two one-link flexible arms," In Proc. IEEE ICRA, pp.1490-1495, 2000.

[17] T. Endo, F. Matsuno, and H. Kawasaki, "Simple boundary cooperative control of two one-link flexible arms for grasping," IEEE Trans. Autom. Control, vol.54, no.10, pp.2470-2476, 2009.

[18] H. Doua and S. Wang, "A boundary control for motion synchronization of a two-manipulator system with a flexible beam," Automatica, vol.50, no.12, pp.3088-3099, 2014.

[19] T.C. Huang, "The Effect of Rotary Inertia and of Shear Deformation on the Frequency and Normal Mode Equations of Uniform Beams With Simple End Conditions," J. Appl. Mech., vol.28, no.4, pp.579-584, 1961.

[20] S.M. Han, H. Benaroya, and T. Wei, "Dynamics of transversely vibrating beams using four engineering theories," J. of Sound and Vibration, vol.225, no.5, pp.935-988, 1999.

[21] A. Soufyane and A. Wehbe, "Uniform stabilization for the Timoshenko beam by a locally distributed damping," Electron. J. Differential Equations, vol.29 pp.1-14, 2003.

[22] Z.H. Luo, N. Kitamura, and B.Z. Guo, "Shear Force Feedback Control of Flexible Robot Arms," IEEE Trans. Robot. Automat., vol.11 pp.760-765, 1995.

[23] Z.H. Luo, "Direct strain feedback control of flexible robot arms: New theoretical and experimental results," IEEE Trans. Autom. Control, vol.38, pp.1610-1622, 1993.

[24] W. Littman and L. Markus, "Stabilization of a Hybrid system of Elasticity by Feedback Boundary Damping," Ann. Mat. Pura Appl., vol.152, no.1, pp.281-330, 1988.

[25] A. Pazy, Semigroups of linear operators and applications to partial differential equations, Springer-Verlag, 1983.

[26] B.Z. Guo, J.M. Wang, and S.P. Yung, "Bounday stabilization of a flexible manipulator with rotational inertia," Differential and Integral Equaations, vol.18, no.9, pp.1013-1038, 2005.

[27] Z. Liu and S. Zheng, Semigroups Associated with Dissipative Systems, Chapman and Hall/CRC, 1999.

[28] K.J. Engel and R. Nagel, One-Parameter Semigroups for Linear Evolution Equations, Springer-Verlag New York Inc., 2000.

[29] J. Liang, Y. Q. Chen, and B. Z. Guo, "A Hybrid Symbolic-Numerical Simulation Method for Some Typical Boundary Control Problems," Simulation, vol.80, pp.635-643, 2004. 